Gut, 1973, 14, 977-982

\title{
Solitary angiodysplastic lesions in the ileocaecal region diagnosed by angiography
}

\author{
G. H. WHITEHOUSE \\ From the Department of Radiodiagnosis, University of Liverpool
}

SUMMARY Single angiodysplasias were demonstrated by preoperative angiography in four patients. In two cases the lesions were in the terminal ileum, and were situated just above the caecum in the other two patients. In three patients, contrast medium was extravasated into the bowel lumen. Histological verification was obtained in three instances. The literature on intestinal angiodysplastic lesions was reviewed, with especial regard to their distribution and radiological manifestations. The relative frequency of angiodysplasias in the right side of the colon is noted. Visceral angiography is an important investigation in cases where there is persistent or recurrent bleeding from the gastrointestinal tract, especially when barium studies and laparatomy have been negative.

No cause for gastrointestinal haemorrhage is found in over half of the patients subjected to full barium investigations and laparotomy (Retzlaff, Hagedorn, and Bartholomew, 1961). The investigation of haemorrhage from the alimentary tract has been considerably helped in recent years by selective angiographic studies of the coeliac and mesenteric arteries. The site of active bleeding as well as the underlying pathology may be demonstrated by this method (Baum, Stein, Nusbaum, and Chait, 1969). Vascular malformations within the gut wall are an infrequent but important cause of gastrointestinal haemorrhage. They are often solitary and may be so small as to elude the most painstaking search at laparotomy, as well as being invisible on barium studies. Coeliac and mesenteric angiography is a useful method for demonstrating these vascular abnormalities.

There is some variation in pathology (Kaijser, 1936) regarding type and size of vessel involved, and there has been some doubt as to whether vascular abnormalities are congenital or neoplastic in origin. This has lead to confusion in terminology. For instance, 'haemangioma', 'telangectasia', and 'arteriovenous malformation' are often regarded as interchangeable and, when used in the strictest pathological sense, are often difficult to differentiate on histological grounds. Although there may be a tendency for enlargement (Packard, 1945), the lesions

Received for publication 29 August 1973. are now generally regarded as being congenital (Shepherd, 1953). The term 'angiodysplasia' is used to encompass these vascular abnormalities and also stresses their congenital nature (Halpern, Turner, and Citron, 1968).

The lesions may be solitary or multiple and diffuse, and occur anywhere in the gastrointestinal tract. However, a review of the literature has revealed that frequent sites of a solitary lesion are the ileum and proximal portion of the colon. Four angiodysplastic lesions will be considered in conjunction with the angiographic findings.

\section{Method}

In cases 1,3 , and 4 the superior mesenteric artery was selectively catheterized by a green Kifa catheter which was introduced from the femoral artery by the Seldinger technique. Twenty-five $\mathrm{ml}$ of Urografin $60 \%$ was injected under a pressure of $4 \mathrm{~g} / \mathrm{cm}^{2}$ from a mechanical injector. The first exposure was made after $5 \mathrm{ml}$ of contrast medium was injected. Films were exposed at a rate of four per second for two seconds, followed by two films per second for a further eight seconds.

In case 2 , the superior mesenteric artery could not be selectively catheterized. Thirty-five ml Urografin $60 \%$ was injected through a straight catheter whose tip was situated in the lumen of the abdominal aorta at the level of the lower part of the twelfth thoracic vertebral body. The exposure sequence was the same as in the other cases. 


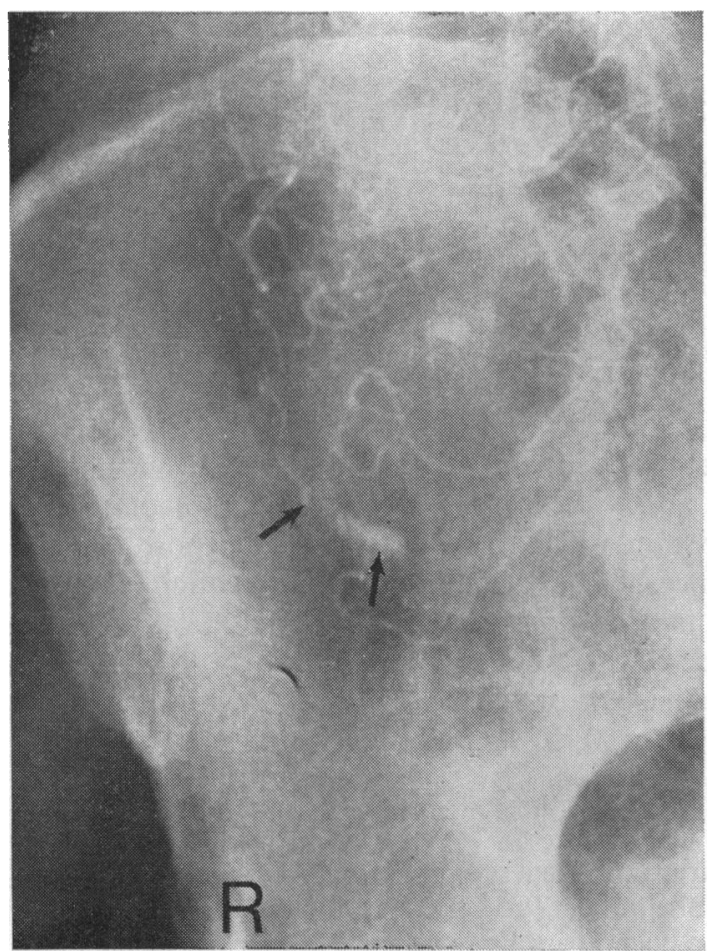

Fig 1

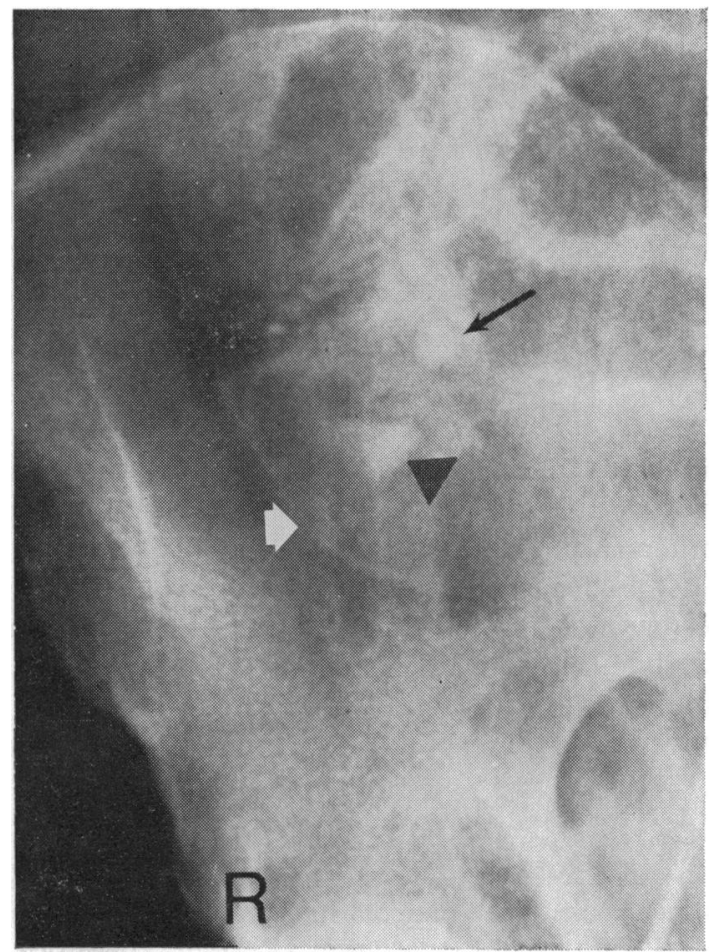

Fig 2

Fig 1 Case 1: superior mesenteric angiogram. Angiodysplasia $(\lambda)$ in caecal region filling in arterial phase.

Fig 2 Case 1: extravasated contrast medium $(\nabla)$ shown in venous phase. Angiodysplasia shown by white arrow. The uppermost opacity $(K)$ is an artefact.

\section{Case 1}

A male aged 65 yr was admitted to hospital with a single episode of frank rectal bleeding and anaemia. A large duodenal diverticulum was shown on a barium meal, and extensive diverticular disease was demonstrated by barium enema. The bleeding arrested spontaneously but a further episode of rectal bleeding occurred two years later. Barium investigations at this time were unchanged from the previous occasion. A superior mesenteric arteriogram (figs 1 and 2) showed an abnormal collection of small blood vessels supplied by the distal branches of the ileocaecal artery and located in the caecum. There was some leakage of contrast medium into the lumen. At laparotomy the same day, no abnormality of the bowel was present on inspection and palpation. Blood was found in the terminal ileum, the mucosa of which was plum coloured, but no bleeding point was found. In view of the angiographic findings, a right hemicolectomy with removal of the terminal portion of ileum was performed. To the naked eye, the mucous membrane of the resected bowel was intact and no abnormality was visible. Histology showed an area of dilated abnormal arterial vessels in the ileum, with an adjacent irregular collagenous mass containing irregularly arranged bundles of smooth muscle and small capillaries, the features being those of an angiodysplasia. The patient has remained well over the succeeding seven months.

\section{Case 2}

A male aged $62 \mathrm{yr}$ presented with a history of tiredness, dyspnoea, and angina of recent onset but no bleeding per rectum. The only physical abnormality was evidence of anaemia. Sigmoidoscopy and full barium studies were normal. A month later he complained of melaena and frank rectal bleeding. Repeat barium studies were again negative. The bleeding ceased spontaneously, but recurred 


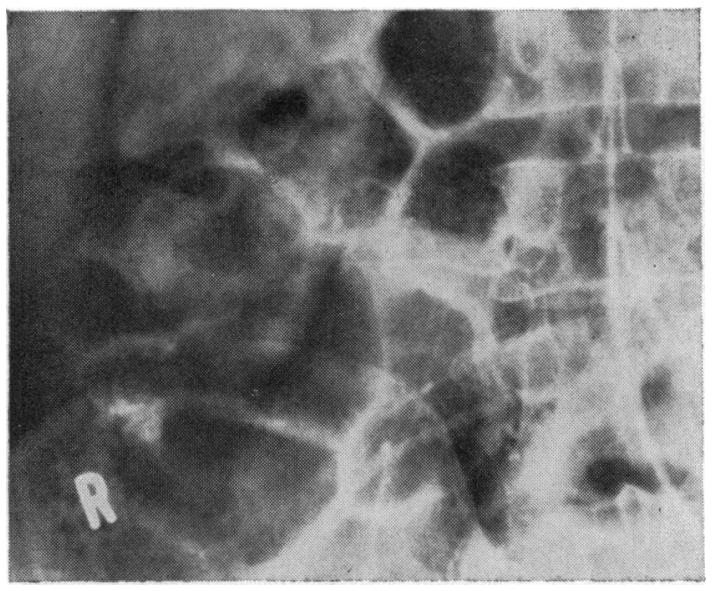

Fig 3 Case 2: aortogram. Angiodysplasia in proximal colon, overlying pelvic brim. Late arterial phase.

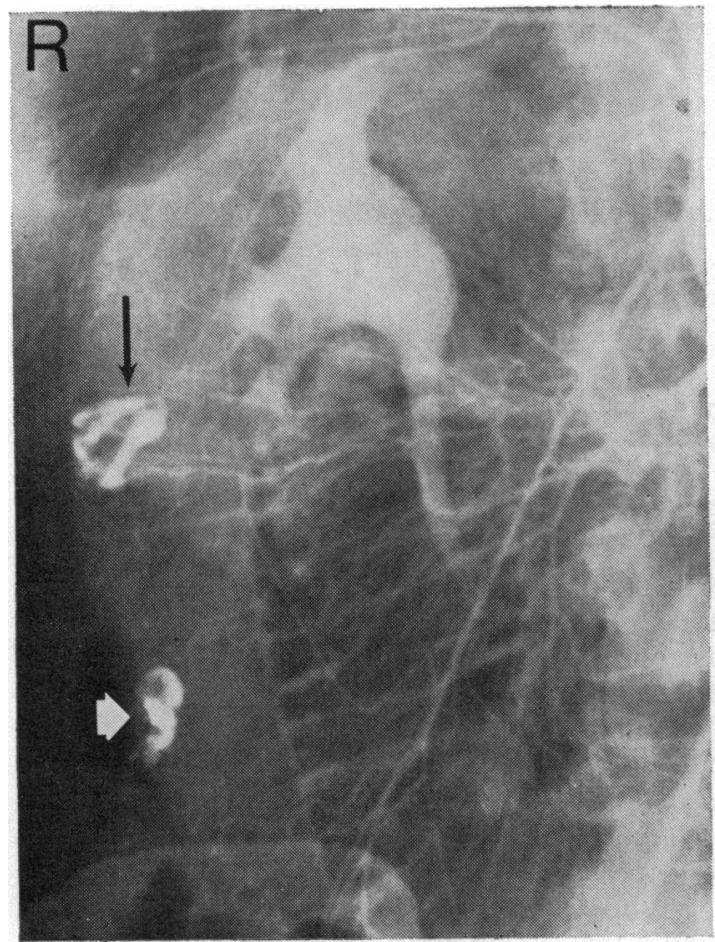

Fig 4 two-and-a-half years later. In view of further negative barium studies and persistence of melaena for 11 days, angiography was performed (fig 3). An abnormal collection of blood vessels supplied by the superior mesenteric artery was seen in the right iliac fossa, together with a large draining vein. At operation the next day, the left half of the colon was filled with blood. A right hemicolectomy with removal of $6 \mathrm{~cm}$ of terminal ileum was performed because of the evidence of angiodysplasia found on angiography. An ulcerated lesion $0.5 \mathrm{~cm}$ in diameter was found $7 \mathrm{~cm}$ distal to the ileocaecal valve, and a collection of thin-walled blood vessels was seen on microscopic examination in the submucosa adjacent to the area of ulceration. Large arterial and venous channels supplied the area, and there was a haematoma in the submucosa. The angiodysplastic area seen on angiography was thus confirmed. The patient has remained well over the ensuing 10 months.

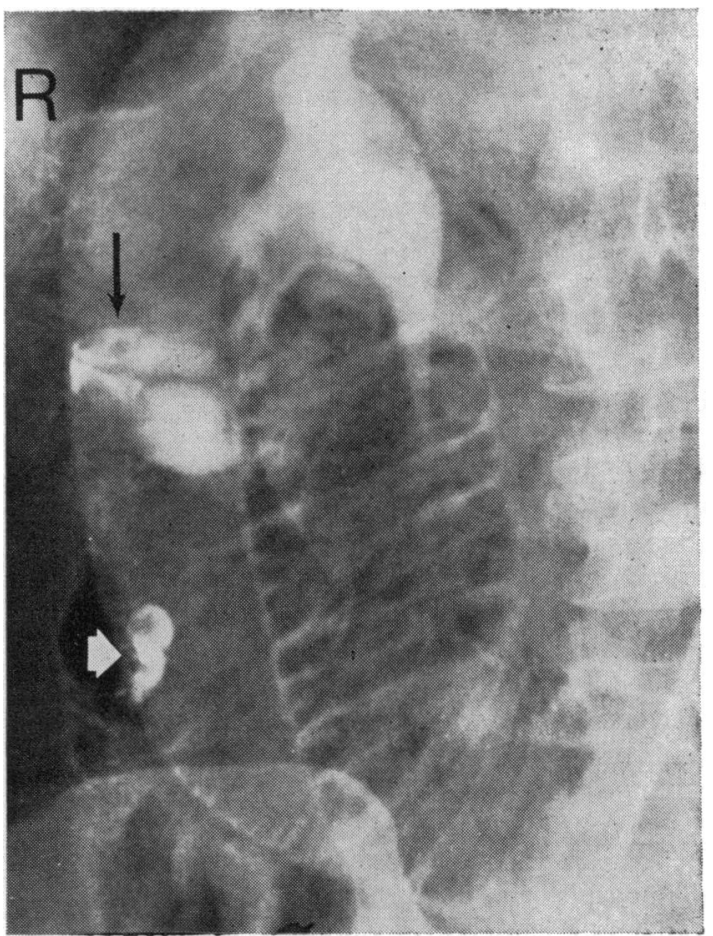

Fig 5

Fig 4 Case 3: superior mesenteric angiogram. Angiodysplasia ( $\downarrow$ ) with large component vessels filling in arterial phase. Lowermost opacity (white arrow) is barium.

Fig 5 Case 3: extravasation of contrast medium with persistent opacification of angiodysplasia in venous 3 * phase. 


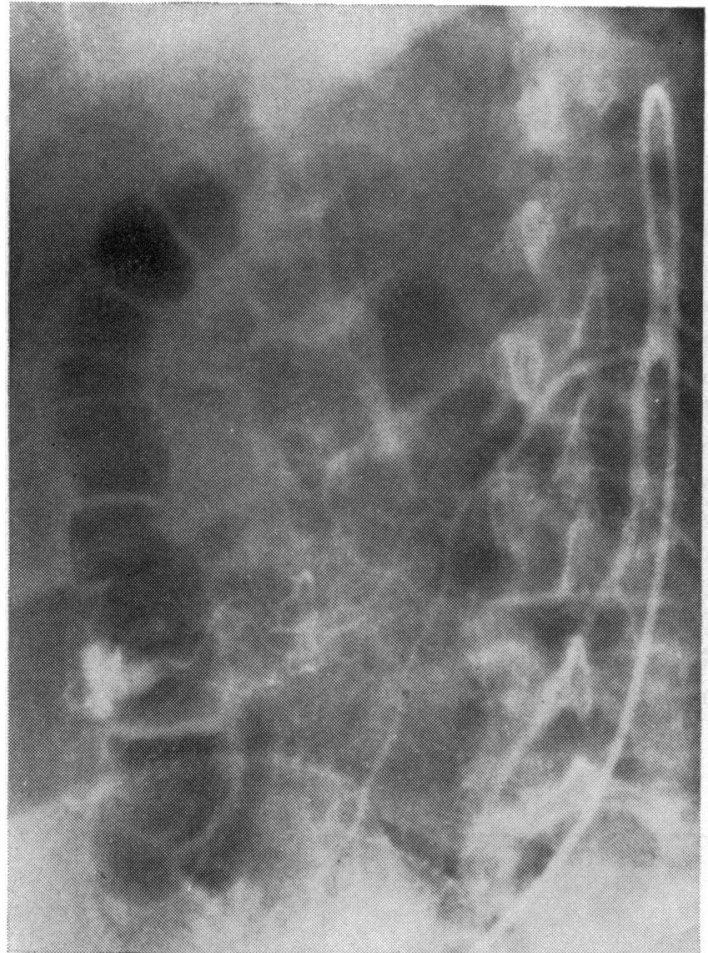

Fig 6

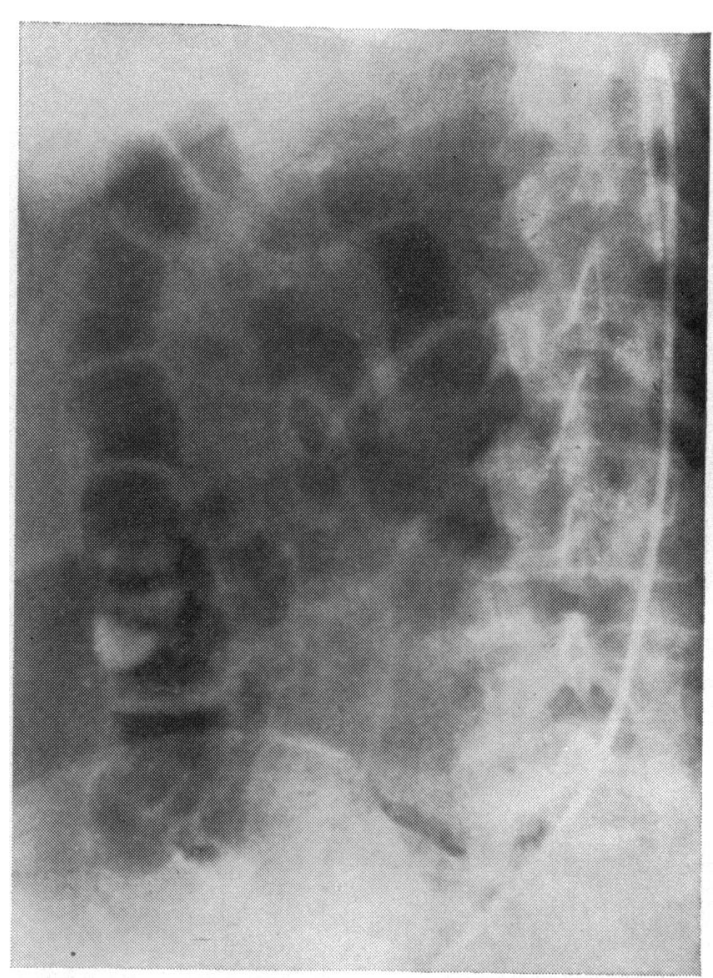

Fig 7

Fig 6 Case 4: Superior mesenteric angiogram, arterial phase. Angiodysplasia in proximal colon with loculation of contrast medium.

Fig 7 Case 4: Loculation persists in late venous phase with extravasation.

\section{Case 3}

A male aged 37 years presented to hospital with a single episode of heavy bleeding per rectum. No abnormality was found at laparatomy. The bleeding ceased spontaneously after the operation, only to recur nine days later. A Gastrografin enema was negative. The second episode of bleeding continued for a week. Superior mesenteric arteriography was then performed (figs 4 and 5) and showed an abnormal collection of vessels of wide and irregular calibre in the terminal ileum. There was extravasation of contrast medium into the bowel lumen, the appearances being those of an actively bleeding angiodysplasia. At laparotomy on the same day, a segment of ileum corresponding to the angiographic abnormality was removed. In the submucosa of the operative specimen, a dilated vein was found to communicate directly with a large but structurally normal artery. The vein opened into the ileum lumen via a breach in the mucosa. The adjacent submucosa consisted of fibrovascular tissue. The patient has been well during the subsequent three years.

\section{Case 4}

A female aged 57 years underwent sigmoidoscopy and barium studies following an episode of torrential haemorrhage and was found to be normal. The bleeding recurred six weeks later and persisted for three days. Apart from moderate anaemia, the patient was otherwise well. A superior mesenteric angiogram (figs 6 and 7) showed some small irregular and tortuous vessels adjacent to a persistently dense loculation of contrast medium and extravasation of contrast medium with the bowel lumen from a point $5 \mathrm{~cm}$ above the caecal pole. The next day a hemicolectomy was performed. There was a blood-filled submucosal space $5 \mathrm{~cm}$ distal to the ileocaecal valve, but no site of mucosal ulceration was present. The radiological appearances, which were characteristic of an angiodysplasia, could not be substantiated by histology. 


\section{Discussion}

Although vascular malformations are uncommon, they are important lesions of the gastrointestinal tract because of diagnostic difficulties and their frequent association with severe and persistent haemorrhage. They may occur at any site from oesophagus to rectum. Symptoms attributable to the angiodysplasias occur during life in $66 \%$ of patients, usually due to bleeding (Gentry, Dockerty, and Clagett, 1949).

In the large series of Gentry et al (1949), $41 \%$ of angiodysplasias were in the small intestine, $31 \%$ in the stomach, and $26 \%$ in the colon. Over 400 cases of small bowel angiodysplasias are described in the world's literature. Only $16 \%$ of lesions in the small intestine are multiple, solitary lesions involving the ileum slightly more often than the jejunum (River, Silverstein, and Tope, 1956). There are 112 reported cases of angiodysplasia of the colon, appendix, and rectum. Within the colon, only $7 \%$ of lesions are multiple or diffuse. Fifty-four per cent are solitary lesions in the sigmoid colon and/or rectum, and $27 \%$ are situated in the caecum (including appendix) or ascending colon. The rest of the single colonic angiodysplasias are in the transverse or descending portions of the colon. The high incidence of vascular abnormalities in the rectum and sigmoid colon may, however, be exaggerated because this region is readily accessible to direct vision. Although bleeding is the commonest clinical presentation, the angiodysplasia may be a mass lesion and cause obstruction (Bailey, Barrick, and
Jenkinson, 1956) or, especially in the small bowel, result in intussusception (Weinstein, Moertel, and Waugh, 1963). The presence of angiodysplastic lesions on the skin or mucous membranes may be associated with similar lesions in the gastrointestinal tract (Halpern et al, 1968). The lesion is discovered at operation in about half the cases, but is often an incidental necropsy finding. Most cases are not symptomatic until the fifth to seventh decades, but may present at any age. The late onset of symptoms is thought to be due to a loss of structural support for the blood vessels resulting from degeneration of connective tissue stroma (Shiffman and Rappaport, 1967).

When cavernous blood spaces are associated with an angiodysplasia, phleboliths may be visible on the plain radiograph. This appearance is most common in the rectal and sigmoid region (Holman, 1948) but may be seen in the small intestine (Marine and Lattomus, 1958). Rarely, a mass lesion may be seen protruding into the lumen of the small intestine (Oschner and Penick, 1957) or large bowel (Stening and Heptinstall, 1970) on barium studies. Most lesions, however, are small and submucosal in situation and are not shown by conventional radiography.

Selective angiography may demonstrate the site of bleeding by virtue of pooling of contrast medium in the bowel lumen at the site of haemorrhage if the rate of blood loss is more than $0.5 \mathrm{ml}$ per minute (Nusbaum, Baum, Blakemore, and Finkelstein, 1965). The nature of the underlying lesion may be identified from its angiographic appearances. Dis-

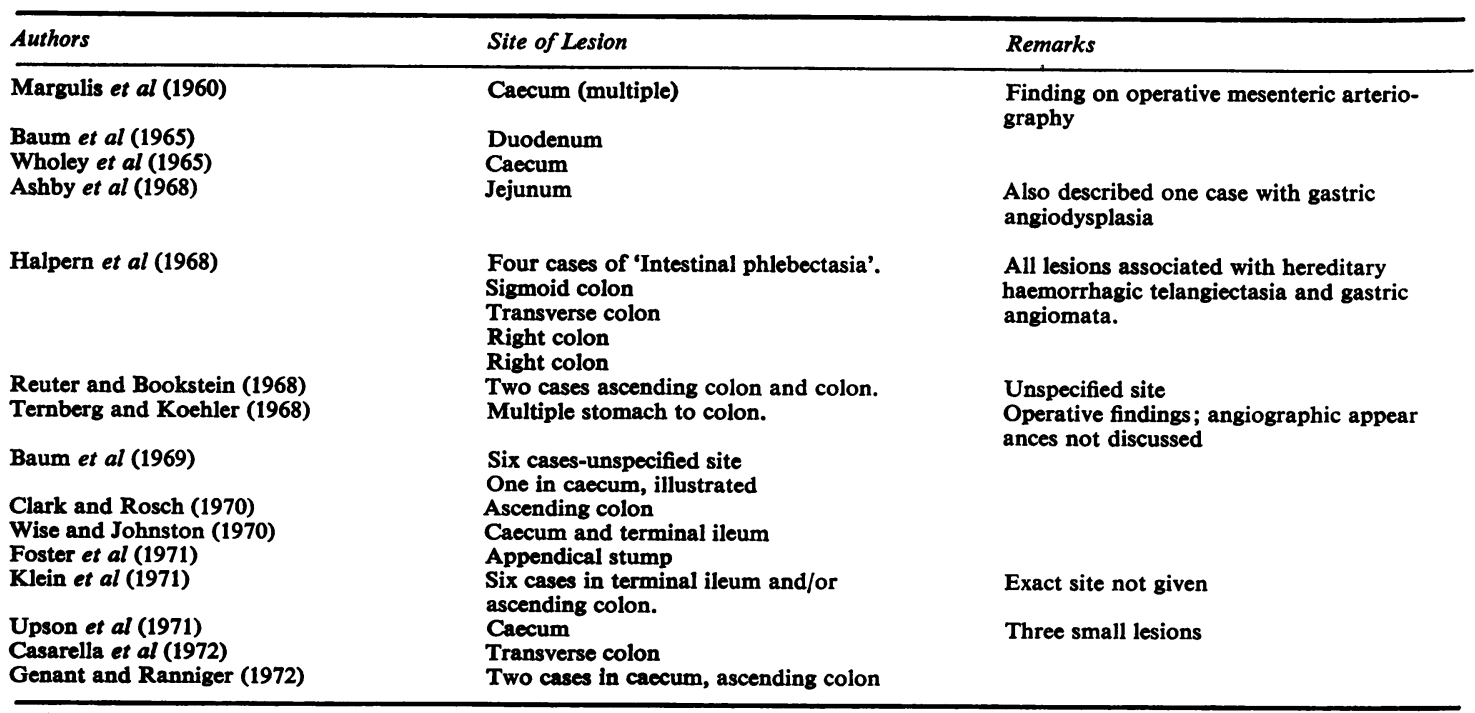

Table Angiographic demonstration of intestinal angiodysplasias 
tinctive vascular patterns, for instance, are associated with neoplasms and inflammatory lesions as well as the angiodysplasias (Baum et al, 1969; Clark and Rosch, 1970; Klein, Alfidi, Meaney, and Poirer, 1971). Angiography has demonstrated intestinal angiodysplasias in 31 reported cases (see table). The typical angiographic appearance of an angiodysplasia is a collection of irregular, dilated, and tortuous small vessels which fill rapidly during the arterial phase, and with persistent opacification and early filling of enlarged draining veins (Klein et al, 1971).

The technique of selective angiography is safe in experienced hands, and the slight morbidity and mortality associated with selective arteriography (Lang, 1963) must be offset against the increased chance of successful localization of the bleeding site at subsequent laparotomy and a shorter operating time (Ashby, Mott, and Starer, 1968). If the patient is in a state of shock from blood loss at the time of angiography, there is a risk of femoral artery thrombosis at the puncture site (Reuter and Bookstein, 1968). It is important that the angiography should not follow barium investigations as the vasculature may be obscured (Baum et al, 1969).

In the present series, all the angiodysplasias were supplied by the superior mesenteric artery, two being situated in the caecum and the other two in the terminal ileum. Three of the lesions were associated with extravasation of contrast medium into the bowel lumen.

I wish to thank Mr R. Helsby and Professor R. Shields for permission to describe patients under their care.

\section{References}

Ashby, E. C., Mott, T. J., and Starer, F. (1968). Severe gastrointestinal haemorrhage: haemangiomata demonstrated by selective visceral arteriography. Brit. med. J., 4, 737-739.

Bailey, J. J., Barrick, C. W., and Jenkinson, E. L. (1956). Hemangioma of the colon. J. Amer. med. Ass., 160, 658-659.

Baum, S., Nusbaum, M., Blakemore, W. S., and Finkelstein, A. K. (1965). The preoperative radiographic demonstration of intraabdominal bleeding from undetermined sites by percutaneous celiac and superior mesenteric arteriography. Surgery, 58, 797-805.

Baum, S., Stein, G. N., Nusbaum, M., and Chait, A. (1969). Selective arteriography in the diagnosis of hemorrhage in the gastrointestinal tract. Radiol. Clin. N. Amer., 7, 131-145.

Casarella, W. J., Kanter, I. E., and Seaman, W. B. (1972). Right sided colonic diverticula as a cause of acute rectal hemorrhage. New Engl. J. Med., 286, 450-453.

Clark, R. A., and Rosch, J. (1970). Arteriography in the diagnosis of large bowel bleeding. Radiology, 94, 83-88.

Foster, J. H., Morgan, C. V., Therlkell, J. B., and Yune, H. Y. (1971). Vascular malformation of the appendiceal stump. J. Amer. med. Ass., 215, 636-638.

Genant, H. K., and Ranniger, K. (1972). Vascular dysplasias of the ascending colon : report of two cases and review of the literature. Amer. J. Roentgenol., 115, 349-354.

Gentry, R. W., Dockerty, M. B., and Clagett, O. T. (1949). Vascular malformations and vascular tumors of the gastrointestinal tract. Int. Abstr. Surg., 88, 281-323.

Halpern, M., Turner, A. F., and Citron, B. P. (1968). Hereditary haemorrhagic telengiectasia. Radiology, 90, 1143-1149.

Holman, C. C. (1948). Haemangioma of the sigmoid colon. Brit. J. Surg., 36, 210.

Kaijser, R. (1936). Uber Hämangioma des Tractus gastrointestinalis. Arch. Klin. Chir., 187, 351-388.

Klein, H. J., Alfidi, R. J., Meaney, T. F., and Poirer, V. C. (1971). Angiography in the diagnosis of chronic gastrointestinal bleeding. Radiology, 98, 83-91.

Lang, E. K. (1963). A survey of complications of percutaneous retrograde arteriography: Seldinger technic. Radiology, 81, 257-263.

Margulis, A. R., Heinbecker, P., and Bernard, H. R. (1960). Operative mesenteric arteriography in the search for the site of bleeding in unexplained gastrointestinal haemorrhage. Surgery, 48, 534-539.

Marine, R., and Lattomus, W. W. (1958). Cavernous hemangioma of the gastrointestinal tract. Radiology, 70, 860-863.

Nusbaum, M., Baum, S., Blakemore, W. S., and Finkelstein, A. K. (1965). Demonstration of intraabdominal bleeding by selective arteriography. J. Amer. med. Ass., 191, 389-390.

Oschner, S., and Penick, R. M., Jr. (1957). Hemangioma of the small intestine. Radiology, 68, 845-848.

Packard, G. B. (1945). Hemangioma of the intestine. Amer. J. Surg., 67, 556-562.

Retzlaff, J. A., Hagedorn, A. B., and Bartholomew, L. G. (1961). Abdominal exploration for gastrointestinal bleeding of obscure origin. J. Amer. med. Ass., 177, 104-107.

Reuter, S. R., and Bookstein, J. J. (1968). Angiographic localization of gastrointestinal bleeding. Gastroenterology, 54, 876-883.

River, L., Silverstein, J., and Tope, J. W. (1956). Benign neoplasms of the small intestine: a critical comprehensive review with reports of 20 new cases. Int. Abstr. Surg., 102, 1-38.

Shepherd, J. A. (1953). Angiomatous conditions of the gastrointestinal tract. Brit. J. Surg., 40, 409-421.

Shiffman, M. A., and Rappaport, I. (1967). Multiple phlebectasia. Arch. Surg., 94, 771-775.

Stening, S. G., and Heptinstall, D. P. (1970). Diffuse cavernous haemangioma of the rectum and sigmoid colon. Brit.J. Surg., 57, 186-189.

Ternberg, J. L., and Koehler, P. R. (1968). The use of arteriography in the diagnosis of the origin of acute gastrointestinal hemorrhage in children. Surgery, 63, 686-689.

Upson, J. F., Bunnell, I., and Kokkinopoulis, E. (1971). Hemangioma of the cecum: diagnosis by angiography. J. Amer. med. Ass., 217, 1104-1105.

Weinstein, E. C., Moertel, C. G., and Waugh, J. M. (1963). Intussuscepting hemangiomas of the gastrointestinal tract. Ann. Surg., 157, 265-270.

Wholey, M. H., Bron, K. M., and Haller, J. D. (1965). Selective angiography of the colon. Surg. Clin. N. Amer., 45, 1283-1291.

Wise, R. E., and Johnston, D. O. (1970). The role of angiography in the evaluation of surgical patients. Surg. clin. N. Amer., 50, 645-662. 